\title{
Monitoreo de la aplicación de vinaza como fertilizante en caña de azúcar con indicadores microbianos de suelo
}

\section{Monitoring the application of vinasse as fertilizer of sugarcane with soil microbial indicators}

Senatore, Daniella (1); Queirolo, Agustina (1); Wajswol, Sergio (1); Bajsa, Natalia (1).

(1) Laboratorio de Ecología Microbiana, Departamento de Bioquímica y Genómica Microbianas, Instituto de Investigaciones Biológicas Clemente Estable (IIBCE), Uruguay.

Contacto: nbajsa@iibce.edu.uy

RECIBIDO: 6/4/2017 - APROBADO: 20/6/2017

\begin{abstract}
Resumen
En la última década se ha experimentado un significativo cambio en el sistema energético mundial hacia el uso de biocombustibles. En Uruguay, el bioetanol a partir de caña de azúcar se convirtió desde 2005 en una importante fuente de energía alternativa. Durante su producción se generan de 8 a 13 litros de vinaza por litro, residuo líquido rico en materia orgánica, potasio, calcio, magnesio, azufre y nitrógeno. Su uso como fertilizante es una alternativa que permite su degradación y el reciclaje de sus nutrientes, en sustitución de fertilizantes químicos. Con el objetivo de identificar indicadores para el monitoreo de la aplicación de vinaza al cultivo de caña de azúcar, se evaluaron diferentes parámetros microbianos del suelo en predios de producción de Bella Unión, Artigas, Uruguay. Se estableció una línea de base para estas variables y su relación con variables químicas. Por dos años consecutivos se determinó la abundancia de bacterias heterótrofas, amonificantes, actinobacterias, hongos y levaduras, y la actividad microbiana medida como respiración. Las primeras cuatro poblaciones se correlacionaron entre sí y con el contenido de potasio en el suelo. El seguimiento de la aplicación de vinaza permite establecer un protocolo que optimice su aprovechamiento como fertilizante y minimice su impacto ambiental.

Palabras clave: Fertirriego, residuos industriales, mejoramiento de suelos, calidad de suelo, biocombustibles.
\end{abstract}

\begin{abstract}
In the last decade, there has been a significant change in the world energy system towards the use of biofuels. In Uruguay, since 2005 bioethanol from sugar cane has become an important source of alternative energy. During its production, eight to thirteen liters of vinasse are produced per liter, a liquid residue rich in organic matter, potassium, calcium, magnesium, sulfur and nitrogen. Its use as fertilizer is an alternative that allows its degradation and recycling of its nutrients, replacing chemical fertilizers. In order to identify indicators for the monitoring of the application of vinasse to sugarcane culture, different microbial parameters of soil were evaluated in production sites of Bella Unión, Artigas, Uruguay. A baseline was established for these variables and their relationship with chemical variables. For two consecutive years, the abundance of heterotrophic bacteria, ammonifiers, actinobacteria, fungi and yeasts was determined, and the microbial activity measured as respiration. The first four populations correlated with each other and with potassium content in the soil. The monitoring of the application of vinasse allows to establish a protocol that optimizes its use as fertilizer and minimizes its environmental impact. Keywords: Fertirrigation, industrial waste, soil improvement, soil quality, biofuels.
\end{abstract}

\section{Introducción}

En la última década se ha experimentado un significativo cambio en el sistema energético mundial, que se ha orientado hacia el uso de biocombustibles debido al aumento en la demanda de energía a nivel mundial y a las consecuencias del uso de combustibles fósiles. La producción de bioetanol a partir de caña de azúcar se está convirtiendo cada vez más en una importante fuente de energía alternativa (Braga do Carmo, et al., 2013).

En Uruguay, en el año 2005 ALUR S.A. (Alcoholes del Uruguay S.A.) retomó el proyecto sucroalcoholero en la localidad de Bella Unión. En el año 2007 se aprobó la Ley Nº 18.195 que promueve la producción de agrocombustibles y establece que ANCAP (Administración Nacional de Combustibles, Alcohol y Portland) debe incorporar a las gasolinas etanol 
producido en Uruguay y con materia prima de la producción agropecuaria nacional (Uruguay, 2007).

La expansión del sector bioenergético está dando lugar a un aumento en la cantidad de residuos producidos, por lo que la posibilidad de reciclar los productos de desechos que se originen aportará a su sustentabilidad. Estos desechos pueden contener grandes cantidades de $\mathrm{C}$ orgánico y una amplia gama de elementos nutritivos, lo que los hace muy atractivos para ser utilizados como enmiendas o fertilizantes (Cayuela, et al., 2010; Galvez, et al., 2012).

Durante la producción de etanol a partir de la caña de azúcar se genera vinaza, un residuo líquido rico en materia orgánica, potasio, calcio, magnesio, azufre y nitrógeno, cuyo pH varía entre 3,5 y 5 (España-Gamboa, et al., 2012). Por cada litro de etanol producido se obtienen entre 8 y 13 litros de vinaza que no pueden ser vertidos a cursos de agua sin tratamiento previo. Debido a su origen y composición, el uso de la vinaza como fertilizante de cultivos es una alternativa que permite su degradación y el reciclaje de sus nutrientes, en sustitución de fertilizantes químicos (García y Rojas, 2008; Navarro, et al., 2000; de Oliveira, et al., 2009).

Las prácticas agrícolas como el cultivo intensivo, el laboreo y la eliminación de los residuos vegetales han contribuido al agotamiento de las reservas de materia orgánica de los suelos, por lo que la aplicación de materiales como la vinaza puede ayudar a reponer la materia orgánica y mejorar la estructura y fertilidad de los suelos (Bustamante, et al., 2010). El riego con vinaza es una práctica bastante difundida en regiones de cultivo de caña de azúcar en Brasil, donde se han obtenido resultados satisfactorios en relación a la materia orgánica, $\mathrm{pH}$ y nutrientes para el cultivo (Bebé, et al., 2009). En Uruguay, ALUR ha comenzado en 2012 con la aplicación de vinaza al suelo, actividad actualmente autorizada por la DINAMA (Dirección Nacional de Medio Ambiente), sujeta a la realización de estudios y al monitoreo de sus efectos sobre el ambiente.

En la mayoría de los trabajos publicados se evalúa el efecto de la utilización de vinaza analizando el impacto sobre las propiedades fisicoquímicas del suelo y la productividad del cultivo. Sin embargo, son los microrganismos del suelo los responsables de su degradación y pueden ser afectados por su aplicación. Los microorganismos del suelo juegan un rol fundamental en los ciclos biogeoquímicos, contribuyen a la nutrición y salud de las plantas, estructura y fertilidad del suelo, y son esenciales para la productividad y estabilidad de este ecosistema (Kennedy, 1999).

Los parámetros microbiológicos son indicadores potenciales de la calidad de suelo, por su mayor sensibilidad para detectar cambios que las propiedades físicas y químicas. El principal desafío actual consiste en interpretar valores aislados de estos indicadores, dado que no existen valores de referencia para la comparación, a diferencia de los indicadores químicos de fertilidad para los cuales sí hay valores de referencia disponibles. Por este motivo, para interpretar indicadores microbiológicos en general se emplean comparaciones con un control. Otra alternativa es utilizar la variación temporal para monitorear estos bioindicadores y evaluar tendencias con el tiempo (evaluación dinámica) (de Castro Lopes, et al., 2012). Una gran cantidad de métodos han sido sugeridos como indicadores de calidad de suelo. Algunos intentos de seleccionar y ranquear una serie de técnicas llegaron a la elaboración de listas de indicadores basados en medidas de diversidad y funciones ecológicas realizadas por técnicas clásicas o moleculares, dando importancia al lugar y escala del estudio (Ritz, et al., 2009; Stone, et al., 2016).
Las consecuencias de la aplicación de vinaza pueden depender de muchos factores relacionados al tipo de suelo, el cultivo y su manejo y a las características de la vinaza, por lo que es fundamental la investigación local sobre los efectos de la aplicación de este efluente.

Con el objetivo de identificar indicadores para el monitoreo de la aplicación de vinaza, en este trabajo se evaluaron diferentes parámetros microbianos del suelo en predios de producción de caña de azúcar. Se estableció una línea de base para estas variables y su relación con parámetros químicos del suelo.

\section{Materiales y Métodos}

\section{Obtención de muestras de suelo}

Las muestras de suelo se tomaron en predios de productores de caña de azúcar ubicados en las cercanías de la destilería de ALUR, Bella Unión, departamento de Artigas, Uruguay $\left(30^{\circ} 19^{\prime} \mathrm{S}\right.$ y $\left.57^{\circ} 36^{\prime} \mathrm{W}\right)$. La región presenta un clima subtropical húmedo, con temperatura media anual de $19,6^{\circ} \mathrm{C}$, máxima mensual promedio de $26,2^{\circ} \mathrm{C}$ y mínima de $13,7^{\circ} \mathrm{C}$. La precipitación es de $1366 \mathrm{~mm}$ al año, menor en agosto, con $66 \mathrm{~mm}$, y mayor en abril, con un promedio de $147 \mathrm{~mm}$. Los predios de producción de caña de azúcar han integrado un complejo agroindustrial por décadas. En este trabajo se seleccionaron 24 chacras con diferentes tipos de suelo representativos de la zona, asociados a las siguientes unidades geológicas: L1, L2, LB2, F2, LF2, S1 (L: Libertad, B: Basalto, F: Fray Bentos, S: Salto), según la carta de suelos. Las mismas han recibido aplicaciones anuales de vinaza durante 5-6 años, en una dosis promedio de $100-150 \mathrm{~m}^{3} /$ ha (Figura 1 ).

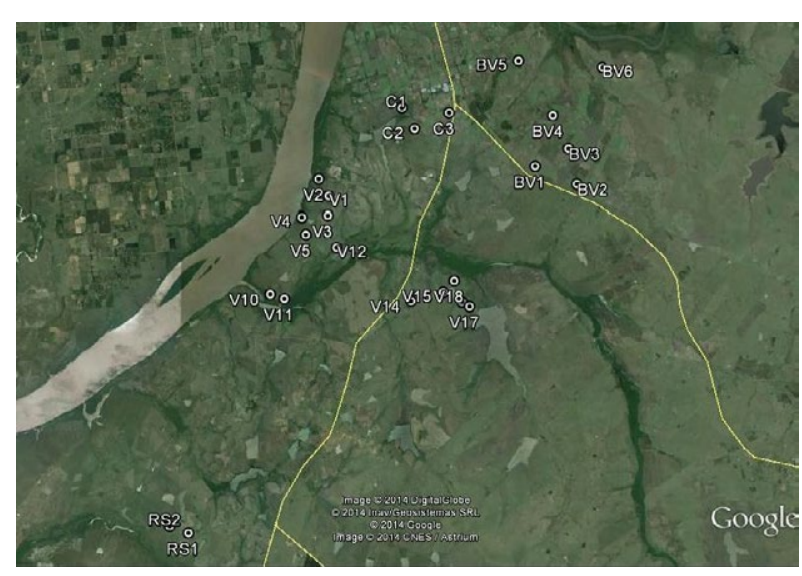

Figura 1. Ubicación de los sitios seleccionados para el monitoreo de la aplicación de vinaza en predios de producción de caña de azúcar en Bella Unión, Artigas.

En mayo de 2015 y junio de 2016 se tomaron muestras de suelo antes de la cosecha y de la aplicación anual de vinaza. Se realizaron muestras compuestas por 10 tomas de suelo al azar de cada predio, con un calador de $2 \mathrm{~cm}$ de diámetro y de 0 a $10 \mathrm{~cm}$ de profundidad, a partir de la base de los camellones. Las muestras se secaron al aire a $20^{\circ} \mathrm{C}$, en los casos que fue necesario para ajustar la humedad, y se tamizaron por malla de $2 \mathrm{~mm}$ para su homogenización. Se conservaron a $4{ }^{\circ} \mathrm{C}$ hasta el momento de su análisis. 
En la misma época en la que se tomaron las muestras para este trabajo, el Departamento de Suelos y Aguas (Facultad de Agronomía, UdelaR) midió en cada parcela de producción los siguientes datos de suelo que fueron considerados en la investigación: textura del suelo, $\mathrm{pH}$ (al agua), conductividad eléctrica (CE), $\mathrm{P}$ disponible (Bray-1), cationes intercambiables ( $\mathrm{Ca}, \mathrm{Mg}, \mathrm{K}$ y Na) y carbono orgánico.

\section{Cuantificación de microorganismos}

En todas las muestras se determinó el número de microorganismos cultivables pertenecientes a diferentes grupos. Para esto se suspendieron $5 \mathrm{~g}$ de suelo en $45 \mathrm{~mL}$ de pirofosfato de sodio $0,1 \%(\mathrm{p} / \mathrm{v})$ estéril y se agitaron a $150 \mathrm{rpm}$ durante 30 $\mathrm{min}$. Se realizaron diluciones seriadas que fueron sembradas en diferentes medios semiselectivos. Se utilizó medio Tryptic Soy Agar 1/10 (TSA 1/10) para bacterias heterótrofas aerobias (Smit, et al., 2001) y medio Starch Casein Agar (SCA) para actinobacterias (Leoni y Ghini, 2003) suplementados con cicloheximida $100 \mu \mathrm{g} / \mathrm{ml}$. El medio Agar Malta (AM) 2\%, suplementado con cloranfenicol $100 \mu \mathrm{g} / \mathrm{ml}$ y ajustado el $\mathrm{pH}$ a 4,5 con ácido láctico, se utilizó para recuento de hongos filamentosos y levaduras (Bettucci y Roquebert, 1995). Las placas se incubaron a $25^{\circ} \mathrm{C}$ y se determinó el número de colonias características a las $72 \mathrm{~h}$ o 7 días, para hongos y levaduras o bacterias heterótrofas y actinobacterias, respectivamente.

Para cuantificar bacterias amonificantes se utilizó la técnica del número más probable (NMP) en medio con peptona $4 \%(\mathrm{p} / \mathrm{v})$. Los tubos se incubaron por $48 \mathrm{~h}$ a $150 \mathrm{rpm}$ y $25^{\circ} \mathrm{C}$ y se determinó la producción de amonio utilizando el reactivo de Nessler (Seeley, et al., 1991).

\section{Medida de actividad microbiana por respirometría}

En frascos herméticamente cerrados se incubaron $25 \mathrm{~g}$ de suelo a $25^{\circ} \mathrm{C}$ por $48 \mathrm{~h}$, previo ajuste de la humedad al $75 \%$ de la capacidad de campo. La concentración de $\mathrm{CO}_{2}$ se determinó por cromatografía de gases utilizando un cromatógrafo Shimadzu GC14B con detector de conductividad térmica
(TCD) y una columna Alltech CTR I (concéntrica de 6 pulgadas de largo, con la columna exterior de 6’ x 1/4" de tamices moleculares activados, y la columna interior de $6^{\prime} \times 1 / 8^{\prime \prime}$ de una mezcla porosa de polímeros). El detector operó a $60^{\circ} \mathrm{C}$ y $120 \mathrm{~mA}$, la temperatura de la columna fue $60^{\circ} \mathrm{C}$ y el gas carrier He a $40 \mathrm{~mL} / \mathrm{min}$. Como estándar se usó una mezcla gaseosa que contenía $\mathrm{CO}_{2} 2,7$ \% (v/v) (Sparling y West, 1990).

\section{Análisis de correlaciones}

Utilizando el programa Statistica 10.0 se generó una matriz de correlación de Pearson entre las variables microbiológicas estudiadas (valores de abundancia de bacterias y respiración) y variables químicas del suelo. Los datos de abundancia de los grupos microbianos fueron previamente transformados $(\log 10)$. Se utilizaron los valores de las variables para cada año, previamente estandarizados utilizando la fórmula: (valor-media)/desvío estándar. Se consideraron significativas las correlaciones con $\mathrm{p}<0,05$.

\section{Resultados y Discusión}

\section{Medida de parámetros microbiológicos en suelo}

En este trabajo se estableció una línea de base de varios parámetros microbiológicos en suelos sometidos a la aplicación de vinaza como fertilizante de la caña de azúcar. Estos parámetros fueron seleccionados previamente a partir de un grupo mayor de variables medidas en ensayos de campo instalados en la zona en estudio, donde se evaluó el efecto de esta práctica sobre las comunidades microbianas del suelo durante un año. Se había determinado la abundancia de varios grupos mediante técnicas dependientes de cultivo (bacterias heterótrofas, actinobacterias, hongos filamentosos, levaduras y bacterias del ciclo del nitrógeno: amonificantes, nitrificantes y desnitrificantes) y de bacterias por PCR en tiempo real del gen del ARNr 16S. También se midió la actividad y biomasa

\begin{tabular}{|l|c|c|c|c|c|c|}
\hline & Heterótrofas & Amonificantes & Actinobacterias & Hongos & Levaduras & Respiración \\
\hline 2015 & \multicolumn{7}{|c|}{ Abundancia (UFC / g suelo seco) } & mgC-CO $/$ kg suelo \\
\hline Máximo & $5,68 \times 10^{7}$ & $1,10 \times 10^{8}$ & $6,65 \times 10^{6}$ & $2,48 \times 10^{6}$ & $4,28 \times 10^{6}$ & 38,56 \\
Promedio & $3,11 \times 10^{7}$ & $2,19 \times 10^{7}$ & $2,85 \times 10^{6}$ & $1,49 \times 10^{6}$ & $1,07 \times 10^{6}$ & 19,9 \\
Mínimo & $7,06 \times 10^{6}$ & $4,30 \times 10^{6}$ & $7,58 \times 10^{5}$ & $5,51 \times 10^{5}$ & $1,28 \times 10^{5}$ & 8,84 \\
Desvío & $1,80 \times 10^{7}$ & $2,97 \times 10^{7}$ & $1,56 \times 10^{6}$ & $5,04 \times 10^{5}$ & $1,04 \times 10^{6}$ & 8,14 \\
\hline 2016 & \multicolumn{7}{|c|}{} & & & \\
\hline Máximo & $3,37 \times 10^{7}$ & $4,60 \times 10^{6}$ & $6,28 \times 10^{6}$ & $2,88 \times 10^{6}$ & $1,82 \times 10^{7}$ & 5,72 \\
Promedio & $1,38 \times 10^{7}$ & $1,06 \times 10^{6}$ & $2,25 \times 10^{6}$ & $1,42 \times 10^{6}$ & $2,69 \times 10^{6}$ & 2,96 \\
Mínimo & $3,97 \times 10^{6}$ & $9,10 \times 10^{4}$ & $3,57 \times 10^{5}$ & $7,54 \times 10^{5}$ & $7,94 \times 10^{4}$ & 0,36 \\
Desvío & $8,07 \times 10^{6}$ & $1,09 \times 10^{6}$ & $1,43 \times 10^{6}$ & $4,42 \times 10^{6}$ & $3,76 \times 10^{6}$ & 2,20 \\
\hline
\end{tabular}

Tabla 1. Valores de los parámetros microbianos medidos durante el monitoreo de suelos con aplicación de vinaza en otoño de 2015 y 2016. Se presenta el promedio, valores máximo y mínimo y desvío estándar para cada año. 
microbianas por respirometría y el método de fumigación-extracción, respectivamente (Senatore, 2013). Además, se determinaron cambios en la estructura de las comunidades de hongos filamentosos (cultivables) y de bacterias (por DGGE - Denaturing Gradient Gel Electrophoresis- del gen del ARNr 16S). Los parámetros seleccionados para el monitoreo de este estudio fueron aquellos que mostraron diferencias entre los tratamientos con y sin aplicación de vinaza y que son accesibles para el análisis de un gran número de muestras.

En este trabajo el número de microorganismos por gramo de suelo seco determinado para cada grupo se encontró en los siguientes rangos: $10^{6}-10^{7}$ bacterias heterótrofas, $10^{5}-10^{8}$ amonificantes, $10^{5}-10^{6}$ actinobacterias y hongos, $10^{5}-10^{7}$ levaduras (Tabla 1).

En los ensayos realizados previamente en otros suelos de la zona en estudio se encontró una abundancia de bacterias heterótrofas de $10^{7}-10^{8} \mathrm{UFC} / \mathrm{g}$ suelo seco, un poco superior a la determinada en estas chacras (Senatore, 2013). En India se hallaron valores promedio de $8 \times 10^{8} \mathrm{UFC} / \mathrm{g}$ de suelo para las bacterias heterótrofas en suelos cultivados con caña de azúcar (Shukla, et al., 2008). Las poblaciones de actinobacterias registradas en los ensayos previos fueron también superiores, del orden $10^{6}-10^{7} \mathrm{UFC} / \mathrm{g}$ de suelo seco (Senatore, 2013). Estudios realizados en India en suelos cultivados con caña de azúcar reportaron valores promedio para estas poblaciones de $5 \times 10^{3}$ UFC/g de suelo. En Colombia en dos suelos cultivados con maíz se hallaron valores promedio de $3,4 \times 10^{4} \mathrm{y}$ $8,0 \times 10^{4} \mathrm{UFC} / \mathrm{g}$ de suelo (Shukla, et al., 2008; Montenegro, 2008). Previamente, la abundancia de bacterias amonificantes encontrada en los ensayos de la misma zona fue de $10^{6}$ a $10^{7}$ UFC/g suelo y en suelos no rizosféricos utilizados para cultivo de trigo se encontraron $1,8 \times 10^{6}$ células por gramo de suelo, similar al rango obtenido en este trabajo (Carrillo, 2003; Senatore, 2013). La abundancia de hongos filamentosos en los ensayos mostró valores del orden de $10^{5}$ y $10^{4} \mathrm{UFC} / \mathrm{g}$ de suelo, mientras que en suelos cultivados con caña de azúcar se reportaron valores promedio de 5,8 x 103 $\mathrm{UFC/g}$ de suelo seco (Fernández, 2012; Shukla, et al., 2008). Las poblaciones de levaduras determinadas previamente en los ensayos de Bella Unión fueron menores, pero los valores de respiración se mantuvieron en el mismo rango que los medidos (Senatore, 2013). Factores como diferencias en las características del suelo, época del año y medotología empleada pueden explicar las diferencias observadas.

\section{Relación entre parámetros microbianos y químicos}

Se realizó un análisis de correlaciones entre las variables microbianas medidas en este estudio y variables químicas del suelo determinadas simultáneamente en los mismos sitios. Las poblaciones de bacterias heterótrofas y amonificantes, actinobacterias y hongos se correlacionaron positivamente entre sí, en ambos años de muestreo, y con la cantidad de $\mathrm{K}^{+}$en el año $2016(p<0.05)$. La vinaza es un residuo especialmente rico en potasio que puede utilizarse en sustitución del potasio químico, y por su aplicación han sido observadas alteraciones en otras bases del suelo (especialmente $\mathrm{Na}^{+}$). La población de bacterias heterótrofas mostró correlación positiva además con $\mathrm{Ca}^{2+} \mathrm{y} \mathrm{Na}^{+}$y la de actinobacterias con las cuatro bases. En el año 2015 la respiración se correlacionó con la concentración de $\mathrm{Mg}^{2+} \mathrm{y} \mathrm{Na}^{+}$. Estos resultados pueden indicar que los sitios que recibieron más vinaza y aporte de $\mathrm{K}^{+}$a través de la vinaza presentan mayor cantidad de microorganismos. En los ensayos realizados previamente estos parámetros microbianos aumentaron con el agregado de vinaza y en forma proporcional a la dosis aplicada. En este trabajo las medidas de abundancia y respiración microbiana no se correlacionaron. En los ensayos de campo analizados previamente el agregado de vinaza aumentó la presencia o actividad microbianas dependiendo del tipo de suelo (Senatore, 2013). Si la aplicación de materia orgánica se realiza en un suelo con limitación de otros nutrientes (por ejemplo, N, P, S), no se transforma en biomasa, sino que debido a la actividad microbiana es liberada como $\mathrm{CO}_{2}$ junto con materia orgánica del propio suelo. Con este fenómeno conocido como "priming effect" la materia orgánica no es aprovechada como mejorador de suelo sino que es liberada a la atmósfera (Marstorp, 1996; Bernal, et al., 1998; Bernal, et al., 1998).

De confirmarse la correlación observada entre algunas de las variables microbianas, se podría reducir el número de parámetros a determinar para el seguimiento de los efectos de esta práctica agrícola.

\section{Efecto de la aplicación de vinaza sobre los microorganismos del suelo}

Los efectos de la aplicación de vinaza sobre los microorganismos del suelo que han sido determinados en Uruguay mostraron que son dependientes de la dosis aplicada y del tipo de suelo. En uno de los sitios estudiados previamente la vinaza favoreció la proliferación de bacterias heterótrofas, amonificantes, actinobacterias y hongos filamentosos, mientras que en el otro disminuyó la abundancia de levaduras y aumentó la actividad microbiana. Los efectos de una sola aplicación de vinaza resultaron temporales, revirtiéndose luego de uno o varios meses, dependiendo de la variable analizada (Senatore, 2013).

En general se ha reportado que la vinaza provoca un aumento de la biomasa y de la actividad microbianas (Sanomiya, et al., 2006; Passarin, et al., 2007; Montenegro, 2008) sin alterar la calidad de la caña producida (Co Júnior, et al., 2008). En estudios realizados por Orlando (1983) se observó una alta proliferación de microorganismos en suelos tratados con vinaza hasta los 30 días después de la aplicación, y se registró luego una tendencia descendente de las poblaciones microbianas. En India se llevaron a cabo otros estudios en los que se adicionaba a suelos agrícolas lodos del tratamiento de residuos de destilerías; en estos casos se observaron aumentos en el número de bacterias heterótrofas para diferentes cantidades aplicadas (Tripathi, 2011).

A su vez, estudios realizados en praderas de Austria que recibieron aplicaciones de lodos provenientes de ganado mostraron un aumento significativo de la biomasa microbiana y de los niveles de mineralización de N (Kandeler, et al., 1994). El incremento de las bacterias amonificantes como consecuencia de aplicar residuos con alta carga de materia orgánica sugiere una transformación del $\mathrm{N}$ orgánico a inorgánico aumentando la disponibilidad de este nutriente para las plantas. En otro trabajo se observó que la aplicación de vinaza altera la diversidad de las bacterias naturalmente presentes en el suelo. Los Actinomycetales fueron detectados más frecuentemente en suelos donde se aplicó vinaza, lo que indica que este tipo de microorganismos se ve favorecido por este manejo (Pine Omori, et al., 2016).

Profundizando en la integración de la información sobre los sitios, formas de manejo, rendimiento del cultivo 
y características de la vinaza aplicada, se podrá mejorar el seguimiento de esta práctica agrícola y establecer un protocolo que optimice su aprovechamiento como fertilizante y minimice su impacto ambiental. Para esto se torna necesario un seguimiento de los efectos de su aplicación a través de indicadores microbianos como los utilizados en este estudio, y el establecimiento de valores de referencia a lo largo del tiempo y en otros sistemas para poder validarlos.

\section{Reconocimientos}

Fuentes de financiación: ALUR (Alcoholes del Uruguay S.A.), ANII (Agencia Nacional de Investigación en Innovación), PEDECIBA (Programa de Desarrollo de las Ciencias Básicas).

\section{Referencias}

Bebé, F.V., Rolim, M.M., Pedrosa, E.M.R., Silva, G.B. y Oliveira, V.S., 2009. Avaliação de solos sob diferentes períodos de aplicação com vinhaça. En: Revista Brasileira de Engenharia Agrícola e Ambiental, 13, pp.781-787.

Bernal, M.P., Navarro, A.F., Sánchez-Monedero, M.A., Roig, A. y Cegarra J., 1998. Influence of sewage sludge compost stability and maturity on carbon and nitrogen mineralization in soil. En: Soil Biology and Biochemistry, 30, pp.305-313.

Bernal, M.P., Sánchez-Monedero, M.A., Paredes, C. y Roig A., 1998. Carbon mineralization from organic wastes at different composting stages during their incubation with soil. En: Agriculture, Ecosystems and Environment, 69, pp.175-189.

Bettucci, L. y Roquebert, M.F., 1995. Microfungi from a tropical rain forest litter and soil: a preliminary study. En: Nova Hedwigia, 61, pp.111-118.

Braga Do Carmo, J., Filoso, S., Zotelli, L.C., de Sousa Neto, E.R., Pitombo, L.M., Duarte-Neto, P.J., Vargas, V.P., Andrade, C.A., Gava, G.J.C., Rossetto, R., Cantarella, H., Neto, A. y Martinelli L.A., 2013. Infield greenhouse gas emissions from sugarcane soils in Brazil: effects from synthetic and organic fertilizer application and crop trash accumulation. En: Global Change Biology Bioenergy, 5, pp.267-280.

Bustamante, M.A., Said-Pullicino, D., Paredes, C., Cecilia, J.A. y Moral, R., 2010. Influences of winery-distillery waste compost stability and soil type on soil carbon dynamics in amended soils. En: Waste Management, 30, pp.1966-1975.

Carrillo, L., 2003. Microbiología agrícola. Salta: Universidad Nacional de Salta. ISBN 987-9381-16-5.

Cayuela, M.L., Oenema, O., Kuikman, P.J., Bakker R.R.J.W. y van Groenigen, J.W., 2010. Bioenergy by-products as soil amendments? Implications for carbon sequestration and greenhouse gas emissions. En: Global Change Biology Bioenergy, 2, pp.201-213.

Có Júnior, C., Marques, M.O. y Tasso Júnior, L.C., 2008. Efeito residual de quatro aplicaçoes anuais de lodo de esgoto e vinhaça na qualidade tecnológica da cana de açucar. En: Revista Engenharia Agrícola, Jaboticabal, 28, pp.196-203.

de Castro Lopes, A.A, Gomes de Sousa, D.M., Montandon Chaer, G., Bueno dos Reis Jr, F., Goedert, W.J. y de Carvalho Mendes, I., 2012. Interpretation of microbial soil indicators as a function of crop yield and organic carbon.
En: Soil Science Society of America Journal, 77, pp.461-472. de Oliveira, E.L., Andrade, L.A de B., de Faria, M.A. y Custódio, T.N., 2009. Vinhaça de alambique e nitrogênio na cana-de-açúcar, em ambiente irrigado e não irrigado. En: Revista Brasileira de Engenharia Agrícola e Ambiental, 13, pp.694-699.

España-Gamboa, E.I., Mijangos-Cortés, J.V., HernándezZárate, G., Domínguez Maldonado, J.A. y Alzate-Gaviria, L.M., 2012. Methane production by treating vinasses from hydrous ethanol using a modified UASB reactor. En: Biotechnology for Biofuels, 5(1), pp.82.

Fernández, P., 2012. Efecto de la aplicación de vinaza en cultivos de caña de azúcar sobre la comunidad microbiana del suelo. Montevideo: Facultad de Ciencias. (Tesis de Grado).

Galvez, A., Sinicco, T., Cayuela, M.L., Mingorance, M.D., Fornasier, F. y Mondini C., 2012. Short term effects of bioenergy by-products on soil $\mathrm{C}$ and $\mathrm{N}$ dynamics, nutrient availability and biochemical properties. En: Agriculture, Ecosystems and Environment, 160, pp.3-14.

García, A. y Rojas, C., 2008. Producción de alcohol carburante y uso de sus subproductos en agricultura. En: XI Congreso Ecuatoriano de la Ciencia del Suelo. Quito, Ecuador (29-31 de ${ }^{\circ}$ Ctubre de 2008). Quito: [s.n.].

Kandeler, E., Eder, G. y Sobotik M., 1994. Microbial biomass, $\mathrm{N}$ mineralization, and the activities of various enzymes in relation to nitrate leaching and root distribution in a slurry-amended grassland. En: Biology and Fertility of Soils, 18, pp.7-12.

Kennedy, A.C., 1999. Bacterial diversity in agroecosystems. En: Agriculture, Ecosystems and Environment, 7, pp.65-76.

Leoni, C. y Ghini, R., 2003. Efeito do lodo de esgoto na induçao de supressividade in vitro a Phytophtora nicotianae. En: Fitopatologia Brasileira, 28, pp.67-75.

Marstorp, H., 1996. Influence of soluble carbohydrates, free amino acids and protein content on the decomposition of Lolium multiflorum shoots. En: Biology and Fertility of Soils, 21, pp.257-263.

Montenegro, S.P., 2008. Influencia de la aplicación de vinaza sobre la presencia, actividad y biomasa microbiana del suelo en el cultivo de maíz dulce (Zea mays). Bogotá: Universidad Nacional de Colombia. (Tesis de Maestría).

Navarro, A.R., Sepulveda, M. del C. y Rubio, M.C., 2000. Bioconcentration of vinasse from the alcoholic fermentation of sugar cane molasses. En: Waste Management, 20, pp.581-585.

Orlando Fo, J., 1983. Sistemas de aplicaçao de vinhança em cana de açucar. En: Alcohol e Açucar, 1, pp.28-32.

Passarin, A.L., Rodrigueiro, E.L., Pazzotti Robaina, C.R. y de Conti Medina, C., 2007. Evaulation of aggregate stability in an oxisol trated with different vinasse doses. En: Revista Brasilera de Ciencia do Solo, 31, pp.1255-1260.

Pine Omori, W., Ferreira de Camargo, A., Stropa Goulart, K.C., de Macedo Lemos, E.G. y Marcondes de Souza, J.A., 2016. Influence of vinasse application in the structure and composition of the bacterial community of the soil under sugarcane cultivation. En: International Journal of Microbiology, 2016, 2016, ID 2349514, 11 pp.

Ritz, K., Black, H.I.K., Campbell, C.D., Harris, J.A. y Wood, C., 2009. Selecting biological indicators for monitoring soils: A framework for balancing scientific and technical opinion to assist policy development. En: Ecological Indicators, 9, pp.1212-1221. 
Sanomiya, L.T., Assis, L.C., de Oliveira, J.A. y Nahas, E., 2006. Mineralización de la paja de caña de azúcar en suelo adicionado con viñaza (subproducto de la industria del alcohol de caña de azúcar) y fertilizante nitrogenado. En: Agricultura Técnica (Chile), 66, pp.90-97.

Seeley, H.W., Vandermark, P.J. y Lee, J.J., 1991. Microbes in action: a laboratory manual. New York: W.H. En: Freeman and Company, 4, pp.389-391.

Senatore, D., 2013. Vinaza como fertilizante de caña azucarera: efecto sobre la comunidad bacteriana del suelo. Montevideo: PEDECIBA-UdelaR. (Tesis de Maestría).

Shukla, S.K., Yadav, R.L., Suman, A. y Singh, P.N., 2008. Improving rhizospheric environment and sugarcane ratoon yield through bioagents amended farm yard manure in udic ustochrept soil. En: Soil \& Tillage Research, 99, pp.158-168.

Smit, E., Leeflansg, P., Gommans, S., van den Broek, J., van Mil, S. y Wernars, K., 2001. Diversity and seasonal fluctuations of the dominant members of the bacterial soil community in a wheat field as determined by cultivation and molecular methods. En: Applied and Environmental Microbiology, 67, pp.2284-2291.

Sparling, G.P. y West, A.W., 1990. A comparison of gas chromatography and differential respirometer methods to measure soil respiration and to estimate the soil microbial biomass. En: Pedobiologia, 34, pp.103-112.

Stone, D., Ritz, K, Griffiths, B.G., Orgiazzi, A. y Creamer, R.E., 2016. Selection of biological indicators appropriate for European soil monitoring. En: Applied Soil Ecology, 97, pp.12-22.

Tripathi, B.D., 2011. A short term study on toxic effects of distillery sludge amendment on microbiological and enzymatic properties of agricultural soil in a tropical city. En: Journal of Earth Science and Climate Change, 1, pp.106. Uruguay. Ley 18.195, de 14 de noviembre de 2007. Diario Oficial, 28 de noviembre de 2007, No.27.373 , p.392A . 